\title{
Pharmacokinetics of intravenous ketorolac in cats undergoing gonadectomy
}

\author{
R Villa* , G Ravasio ${ }^{\dagger}$, C Ferraresi ${ }^{*}$, A Zonca*, S Carli*, L Borghi ${ }^{\dagger}$, and P Cagnardi*\$
}

*Università degli Studi di Milano, Dipartimento di Scienze Veterinarie per la Salute, la Produzione Animale e la Sicurezza Alimentare, 20133 Milano, Italy

tUniversità degli Studi di Milano, Dipartimento di Scienze Veterinarie e Sanità Pubblica, 20133 Milano, Italy

SAuthor for correspondence. Email: petra.cagnardi@unimi.it

Supplementary Figure 1. Individual curves of observed and predicted concentrations of ketorolac in serum of 12 cats after I/V administration of ketorolac $(0.5 \mathrm{mg} / \mathrm{kg})$ and pharmacokinetic analysis with a two-compartmental model. Cats 3,6 and 10 are males, all the others are females.

The content of this supplementary information has not been edited. All risk and liability rest with the authors. 


\section{Supplementary Information}
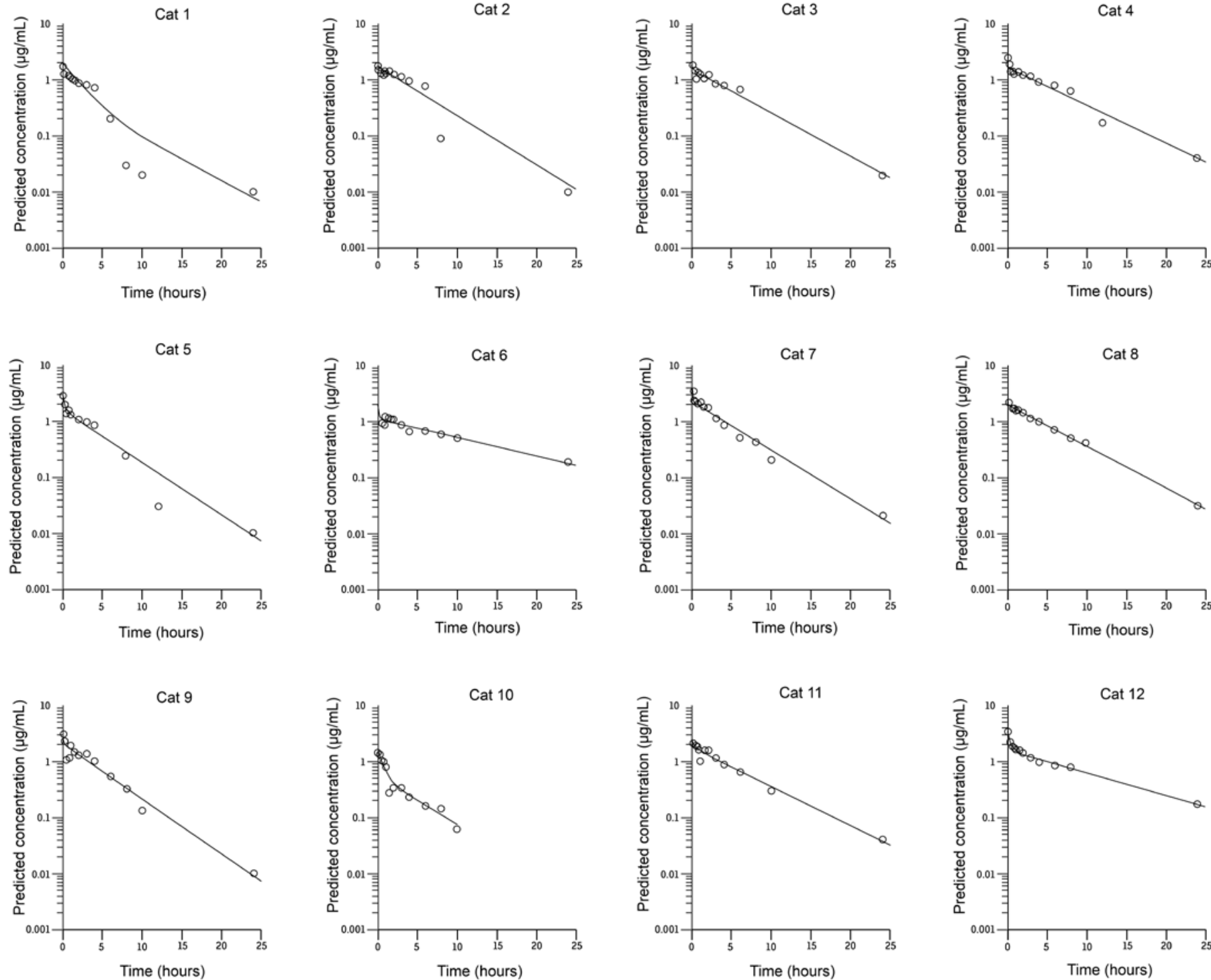